\title{
Probabilistic Approach for Granular Media Equations in the Non Uniformly Convex Case
}

\author{
P. Cattiaux, A. Guillin and F. Malrieu
}

31st October 2018

\begin{abstract}
We use here a particle system to prove a convergence result as well as a deviation inequality for solutions of granular media equation when the confinement potential and the interaction potential are no more uniformly convex. Proof is straightforward, simplifying deeply proofs of Carrillo-McCann-Villani [CMCV03, CMCV06] and completing results of Malrieu [Mal03] in the uniformly convex case. It relies on an uniform propagation of chaos property and a direct control in Wasserstein distance of solutions starting with different initial measures. The deviation inequality is obtained via a $T_{1}$ transportation cost inequality replacing the logarithmic Sobolev inequality which is no more clearly dimension free.
\end{abstract}

Mathematics Subject Classification 2000: 65C35, 35K55, 65C05, 82C22, 26D10, 60E15.

Keywords: Granular media equation, transportation cost inequality, Logarithmic Sobolev Inequalities - Concentration inequalities.

\section{Introduction}

Our main goal will be to deal in a probabilistic way with the following nonlinear equation

$$
\frac{\partial u}{\partial t}=\nabla \cdot[\nabla u+u \nabla V+u \nabla W * u]
$$

where $u(t, x)$ is a time dependent probability measure, $*$ denotes the standard convolution operator and $V$ and $W$ are two convex (at infinity) potentials.

This equation arises (in dimension 1) in the modeling of granular media as follows: consider many infinitesimal particles colliding inelastically. With a correct renormalization between the frequency and the inelasticity of the collisions, $u(t, x)$ turns out to be the velocity of a representative particle (among an infinity). The potential $V$ represents the friction and $W$ the inelastic collisions between particles with different velocities. Note that the particular case $V=0$ and $W(x)=|x|^{3}$ is of special interest and we refer to Benedetto-Caglioti-Pulvirenti [BCP97] for the physical issues (also see [BCCP98]).

Once the problem of existence and uniqueness is tackled, one major problem in this equation is the behavior at infinity: existence of a stationary measure and speed of convergence towards this stationary measure or even distance between two solutions starting at different points. It has been studied in parallel by Carrillo-McCann-Villani [CMCV03, CMCV06] and Malrieu [Mal01, Mal03], under various assumptions on the potentials $V$ and $W$, using analytical and probabilistic approaches respectively (also see [BRV98, BRTV98] for one dimensional particles). We will consider here the probabilistic approach and will recover and generalize slightly results of [CMCV03, CMCV06] as 
well as give a quantified probabilistic approximation of the stationary measure of the granular media equation. It is worthwhile noticing that the analytic methods in [CMCV03, CMCV06] cover a much larger spectrum of non linear p.d.e's (like the porous medium equation), for which the probabilistic approach remains to be written.

As physical interest (and in fact where the main mathematical difficulty resides) dictates the friction term to vanish, we will consider the following two sets of general assumptions:

Definition 1.1 • We say that $W$ satisfies the set of assumptions ( $A$ ) if

A1. the friction term $V=0$;

A2. $W$ is symmetric, i.e. $W(-x)=W(x)$;

A3. $\nabla W$ is locally Lipschitz with polynomial growth, i.e. for some $m$

$$
\forall x, y \in \mathbb{R}^{d},|\nabla W(x)-\nabla W(y)| \leq C(|x-y| \wedge 1)\left(1+|x|^{m}+|y|^{m}\right) .
$$

We also assume that the second derivative of $W$ has a $(m)$ polynomial growth.

A4. $W$ is the sum of a compactly supported $\mathcal{C}^{2}$ function and a $\mathcal{C}^{2}$ uniformly convex function. This last assumption entails some uniform convexity at infinity property, namely that there exist positive $C, \lambda$, such that

$$
\forall x, y, \quad(x-y) \cdot(\nabla W(x)-\nabla W(y)) \geq \lambda\|x-y\|^{2}-C .
$$

- We say that $V$ and $W$ satisfy the set of assumptions ( $A$ ') if $V$ is uniformly convex at infinity (i.e. satisfies (A4)), $W$ is convex at infinity (but not necessarily uniformly) and symmetric, and both satisfy (A3).

As we will see later, these assumptions are not sufficient to get good properties for large time of the granular media equation and will be replaced by some "strict convexity except for a finite number of points" property.

Before further discussing the result, let us first present the probabilistic approach of this problem. The probabilistic interpretation is to consider a Markov process $\left(\bar{X}_{t}\right)_{t \geq 0}$, which law at time $t$ is $u$. It is the solution of the nonlinear S.D.E.

$$
\left\{\begin{array}{l}
d \bar{X}_{t}=\sqrt{2} d B_{t}-\nabla V\left(\bar{X}_{t}\right) d t-\nabla W * u_{t}\left(\bar{X}_{t}\right) d t \\
\mathcal{L}\left(\bar{X}_{t}\right)=u_{t} d x
\end{array}\right.
$$

We wish here first to give sufficient conditions ensuring both existence and ergodicity of the solution of the nonlinear S.D.E. and in a second time to provide a way to simulate this law at each time $t$ with some Gaussian confidence intervals independent of time $t$. These two goals will be carried out through the extensive use of some (linear) particle approximations, i.e. $\left(X_{t}^{N}\right)$ solution of

$$
\begin{cases}d X_{t}^{i, N}=\sqrt{2} d B_{t}^{i}-\nabla V\left(X_{t}^{i, N}\right) d t-\frac{1}{N} \sum_{j=1}^{N} \nabla W\left(X_{t}^{i, N}-X_{t}^{j, N}\right) d t & i=1, \ldots, N \\ X_{0}^{i, N}=X_{0}^{i} & i=1, \ldots, N\end{cases}
$$

If we suppose that $V=0$, we may assume that the center of mass is fixed, and without loss of generality, set to 0 , i.e. $\mathbb{E} \bar{X}_{0}=0$, indeed it is easy to remark that $\bar{X}_{t}-\mathbb{E} \bar{X}_{0}$ satisfies equation (3). This assumption obliges to introduce the projected system onto the set $\sum_{i} x_{i}=0$ (see Section 2), and introduces some intricacies.

Under the strong assumptions of (global) uniform convexity of one of the potentials (the other one being globally convex but non necessarily uniformly), Malrieu [Mal01, Mal03] successively fulfilled these two goals using extensively the so called Bakry-Émery criterion ensuring that a Logarithmic Sobolev inequality holds independently of $N$. He also provided in the same time asymptotic behavior 
in time and concentration inequality, enabling him to recover part of the results of Carrillo-McCannVillani [CMCV03] (see also Bolley-Guillin-Villani [BGV05] for a strengthened deviation inequality for almost quadratic potentials).

The assumption of uniform convexity is however far too strong for applications (preventing for example to consider the case of cubic interaction potential). It can be removed (see [CMCV03]) for a one point degeneracy where the authors obtain various rates of convergence. Another approach is proposed by Carrillo-McCann-Villani in the subsequent [CMCV06] paper introducing tools of contractions in $L^{2}$-Wasserstein distance for length space enabling them to ensure a convergence in Wasserstein distance of the solution of the granular media equation.

We will see here that, with much simpler tools, we can recover their result and may provide also Gaussian confidence bounds for the approximations of the granular media equations. It is worthwhile noticing that, though the invariant measures of the particle system still satisfy some log-Sobolev inequality, it is difficult to obtain dimension-free estimates on the log-Sobolev constants (as for nearest neighbors interactions models in Statistical Mechanics). This prevents us to use this kind of approach.

Our main tool in order to get the Gaussian concentration inequality we need, will thus be a transportation cost-information inequality: let say that $\mu \in T_{1}(C)$ if for every probability measure $\nu$

$$
W_{1}(\nu, \mu) \leq \sqrt{C \operatorname{Ent}(\nu \mid \mu)}
$$

where $W_{1}$ is the usual Wasserstein distance and Ent the Kullback information or relative entropy. In the final section we prove that, under one of the hypothesis (A) or (A'), the law of the particle system at time $t$ satisfies a $T_{1}(C N)$ inequality, for some $C$ that does not depend on $N$ nor on $t$.

To complete the proof of the concentration inequality in the final section we need some "uniform (in time) propagation of chaos". It seems difficult to obtain such a result only assuming (A) or (A'). Indeed if the potentials are non attractive in some (bounded) region, the situation becomes the classical mean-field one where propagation of chaos is controlled on finite time intervals only. That is why we have to reinforce our assumptions.

Our condition below is inspired by the work of Carrillo-McCann-Villani [CMCV06]:

we say that condition $\mathbf{C}(\mathbf{A}, \alpha)$ holds if there exist $A, \alpha>0$ such that for any $0<\epsilon<1$,

$$
\forall x, y \in \mathbb{R}^{d}, \quad(x-y) \cdot(\nabla W(x)-\nabla W(y)) \geq A \epsilon^{\alpha}\left(|x-y|^{2}-\epsilon^{2}\right) .
$$

Remark that this condition implies convexity (if $|x-y| \leq 1 / 2$ choose $\epsilon=|x-y|$, otherwise choose $\epsilon=1 / 4$ ) but is weaker than uniform convexity (which is true with $\alpha=0$ ). Typical examples are polynomial potentials of the form $W(x)=|x|^{2+\alpha}$ which satisfy $\mathbf{C}(\mathbf{A}, \alpha)$ for some positive $A$. In fact this condition is related to the rate at which strict convexity is lost at points.

Condition $\mathbf{C}(\mathbf{A}, \alpha)$ allows us to prove the required "uniform (in time) propagation of chaos" and then to obtain the rate of convergence of $u_{t}$ to the limit $u_{\infty}$ in Wasserstein distance $W_{2}$. These results are obtained in sections 3 and 4.

To complete the description of the paper, the next section contains some useful estimates, and a complete proof of existence and uniqueness for all the equations we have considered ((1) and (3) in particular). The proof is quite natural (using the particle system). We did not find such a proof in the literature except in dimension 1, and as a matter of fact the set of hypotheses we need, slightly differs from the ones used in the analytical literature.

\section{The particle system and solutions of the nonlinear SDE}

In this section we study the particle system and the nonlinear SDE. The first discussion is about case (A) when $V$ vanishes. 
Consider once again the particle system $\left(X_{t}^{N}\right)$ solution of

$$
\begin{cases}d X_{t}^{i, N}=\sqrt{2} d B_{t}^{i}-\nabla V\left(X_{t}^{i, N}\right) d t-\frac{1}{N} \sum_{j=1}^{N} \nabla W\left(X_{t}^{i, N}-X_{t}^{j, N}\right) d t & i=1, \ldots, N \\ X_{0}^{i, N}=X_{0}^{i} & i=1, \ldots, N\end{cases}
$$

Here $X_{0}^{i, N}$ are i.i.d. variables, with common law $\mu_{0}$.

As noted by Malrieu, when $V=0$, the direction $\{(v, \cdot, v)\}$ is quite singular for the particle system, and we then introduce its orthogonal hyperplane $\mathcal{M}=\left\{x \in\left(\mathbb{R}^{d}\right)^{N} ; \sum_{i=1}^{N} x_{i}=0\right\}$, and consider the projected particle system

$$
Y_{t}^{i, N}=X_{t}^{i, N}-\frac{1}{N} \sum_{j=1}^{N} X_{t}^{j, N} \quad \forall i=1, \ldots, N
$$

The process $\left(Y_{t}^{N}\right)_{t \geqslant 0}$ thus verifies the system of SDE's

$$
\begin{cases}d Y_{t}^{i, N}=\sqrt{2} d B_{t}^{i}-\frac{\sqrt{2}}{N} \sum_{j=1}^{N} d B_{t}^{j}-\frac{1}{N} \sum_{j=1}^{N} \nabla W\left(Y_{t}^{i, N}-Y_{t}^{j, N}\right) d t & i=1, \ldots, N \\ Y_{0}^{i, N}=X_{0}^{i}-\frac{1}{N} \sum_{j=1}^{N} X_{0}^{j, N} & i=1, \ldots, N\end{cases}
$$

hence is a diffusion on $\{\mathcal{M}\}$. Note that $\mathbb{E}\left(Y_{t}^{i, N}\right)=0$.

We assume that $W$ is satisfying the assumptions (A) in Definition 1.1. Let us first remark that this new particle system satisfies globally the "convexity at infinity" property. We have to verify, denoting $b(x)=\left(b^{1}(x), \ldots, b^{N}(x)\right), b^{i}$ with values in $\mathbb{R}^{d}$ and

$$
b^{i}(x)=-\frac{1}{N} \sum_{j=1}^{N} \nabla W\left(x_{i}-x_{j}\right)
$$

that condition (2) holds true. Note that, with $\sigma$ the (constant) matrix diffusion coefficient of $\left(Y_{t}^{i, N}\right)_{1_{l} e i \leq N}$, we have $\|\sigma\|_{H S} \leq c$ for some positive $c$, and, as $W$ is symmetric and $x, y \in \mathcal{M}$

$$
\begin{aligned}
(x-y) \cdot(b(x)-b(y)) & =-\frac{1}{2 N} \sum_{i, j=1}^{N}\left(x_{i}-x_{j}-\left(y_{i}-y_{j}\right)\right) \cdot\left(\nabla W\left(x_{i}-x_{j}\right)-\nabla W\left(y_{i}-y_{j}\right)\right) \\
& \leq \frac{1}{2 N} \sum_{i, j=1}^{N}\left(-\lambda\left|x_{i}-x_{j}-y_{i}+y_{j}\right|^{2}+C\right) \\
& =-\lambda\|x-y\|^{2}+\frac{C N}{2},
\end{aligned}
$$

where the latter equality follows as $x, y \in \mathcal{M}$.

This remark allows to prove existence, uniqueness and non-explosion for the solution of the SDE (8). Indeed recall that a sufficient condition for all this to hold is the following: there exists some $\psi$ such that $\psi(x) \rightarrow+\infty$ as $|x| \rightarrow+\infty$ and $\Delta \psi+b . \nabla \psi$ is bounded from above. $\psi$ is a kind of Lyapounov function. Here we may choose $\psi(x)=|x|^{2}$ according to the previous remark applied with $y=0$. In this case non-explosion holds as soon as $\mu_{0}\left(|x|^{2}\right)<+\infty$.

The same argument can be used with (A') if $W$ is everywhere convex.

For the initial system (7) the only thing to show is non explosion. It will follow from Proposition 2.1 below. 


\subsection{Moment controls for the particle system}

In order to prove tightness of the empirical measure of the particle system, and for later use, we first prove some controls on moments.

Proposition 2.1 If $W$ and $V$ satisfy (A'), and $\mu_{0}\left(|x|^{2}\right)<+\infty$ the solution to (7) is non explosive. Furthermore there exists some $K>0$ such that for all $i$,

$$
\sup _{t \geqslant 0} \mathbb{E}\left|X_{t}^{i, N}\right|^{2} \leq \mu_{0}\left(|x|^{2}\right)+K
$$

If $W$ satisfies (A) a similar result holds with $Y_{t}^{i, N}$ in place of $X_{t}^{i, N}$.

\section{Proof}

$\triangleleft$ Using Itô's formula up to the infimum between $t$ and the exit time of a large ball (let $T$ be this infimum) we have

$$
\begin{aligned}
\mathbb{E} \sum_{i=1}^{N}\left|X_{T}^{i, N}\right|^{2}= & \mathbb{E} \sum_{i=1}^{N}\left|X_{0}^{i, N}\right|^{2}+2 d N \mathbb{E}(T)-2 \mathbb{E} \sum_{i=1}^{N} \int_{0}^{T} X_{s}^{i, N} \cdot \nabla V\left(X_{s}^{i, N}\right) d s \\
& -\frac{2}{N} \mathbb{E} \sum_{i, j=1}^{N} \int_{0}^{T} X_{s}^{i, N} \cdot \nabla W\left(X_{s}^{i, N}-X_{s}^{j, N}\right) d s \\
= & \mathbb{E} \sum_{i=1}^{N}\left|X_{0}^{i, N}\right|^{2}+2 d N \mathbb{E}(T)-2 \mathbb{E} \sum_{i=1}^{N} \int_{0}^{T} X_{s}^{i, N} \cdot \nabla V\left(X_{s}^{i, N}\right) d s \\
& -\frac{1}{N} \mathbb{E} \sum_{i, j=1}^{N} \int_{0}^{T}\left(X_{s}^{i, N}-X_{s}^{j, N}\right) \cdot \nabla W\left(X_{s}^{i, N}-X_{s}^{j, N}\right) d s .
\end{aligned}
$$

We may use condition (2), go to the limit with respect to the radius of the ball (hence replace $T$ by $t)$ and obtain the finiteness of the quantity. This implies non explosion.

Furthermore, denoting $v(t)=\mathbb{E} \sum_{i=1}^{N}\left|X_{t}^{i, N}\right|^{2}$, differentiating (see the proof of the next Proposition) and using our conditions (in particular $x . \nabla W(x) \geqslant \lambda_{W}|x|^{2}-C$ ), we get

$$
v^{\prime}(t) \leq-2 \lambda_{V} v(t)+2 d N+3 C N .
$$

Gronwall's lemma and exchangeability conclude the proof in case (A').

The proof is similar for $Y$ in case (A) using the convexity at infinity of the drift, as we previously remarked. $\triangleright$

Proposition 2.2 If $W$ satisfies (A) or $W$ and $V$ satisfy ( $\left.A^{\prime}\right)$, for all $k \in \mathbb{N}$ there exists $C(k)>0$ such that for all $1 \leq i, j \leq N$,

$$
\sup _{t \geqslant 0} \mathbb{E}\left(\left|X_{t}^{i, N}-X_{t}^{j, N}\right|^{2 k}\right) \leq C(k)\left(1+\mu_{0} \otimes \mu_{0}\left(|x-y|^{2 k}\right)\right) .
$$

\section{Proof}

$\triangleleft$ We write the proof in the case $V=0$ (i.e (A)), the case (A') is similar.

Recall that all particles are exchangeable. We may apply Itô's formula up to the exit time of a large ball (for the whole system) and then go to the limit in order to get,

$$
\begin{aligned}
& \mathbb{E} \sum_{i, j=1}^{N}\left|X_{t}^{i, N}-X_{t}^{j, N}\right|^{2 k}=\mathbb{E} \sum_{i, j=1}^{N}\left|X_{0}^{i, N}-X_{0}^{j, N}\right|^{2 k}+2 k(2 k-1) \int_{0}^{t} \mathbb{E} \sum_{i, j=1}^{N}\left|X_{s}^{i, N}-X_{s}^{j, N}\right|^{2 k-2} d s \\
& -\frac{2 k}{N} \mathbb{E} \sum_{i, j, l=1}^{N} \int_{0}^{t}\left(\nabla W\left(X_{s}^{i, N}-X_{s}^{l, N}\right)-\nabla W\left(X_{s}^{i, N}-X_{s}^{l, N}\right)\right) \cdot\left(X_{s}^{j, N}-X_{s}^{l, N}\right)\left|X_{s}^{i, N}-X_{s}^{j, N}\right|^{2 k-2} d s .
\end{aligned}
$$


Denoting $A_{k}(t)=\mathbb{E} \sum_{i, j=1}^{N}\left|X_{t}^{i, N}-X_{t}^{j, N}\right|^{2 k}$, and using (2) we obtain

$$
A_{k}(t) \leq N \mu_{0} \otimes \mu_{0}\left(|x-y|^{2 k}\right)+2 k(2 k-1+C) \int_{0}^{t} A_{k-1}(s) d s-2 k \lambda \int_{0}^{t} A_{k}(s) d s .
$$

Applying Gronwall's lemma and an easy induction we thus have that $A_{k}(t)$ is finite for all $t$. Accordingly we may replace the pair of times $(0, t)$ by $(t, t+\varepsilon)$ and prove that $t \mapsto A_{k}(t)$ is differentiable. Differentiating at time $t$ yields

$$
A_{1}^{\prime}(t) \leq-2 \lambda A_{1}(t)+2 N(d+C) .
$$

Gronwall's lemma yields the desired result for $k=1$. The proof follows, using this bound and an easy induction. $\triangleright$

We are able now to generalize Proposition 2.1 and get uniform moment estimates of every order (under assumptions on the initial condition).

Corollary 2.3 If $W$ satisfies (A) or if $W$ and $V$ satisfy ( $\left.A^{\prime}\right)$ (where $m$ is defined), then for all $t>0$ and all $k \geqslant 1$ there exists a constant $c(k)$ such that for all $i$

$$
\sup _{t \geqslant 0} \mathbb{E}\left|X_{t}^{i, N}\right|^{2 k} \leq c(k)\left(1+\mu_{0}\left(|x|^{2 m k}\right)\right) .
$$

If $W$ satisfies (A) a similar result holds with $Y_{t}^{i, N}$ in place of $X_{t}^{i, N}$.

\section{Proof}

$\triangleleft$ We write the proof under $\left(A^{\prime}\right)$. Let $B_{k}(s)=\mathbb{E}\left(\left|X_{s}^{i, N}\right|^{k}\right)$. As in the previous propositions we shall use Itô's formula up to the stopping time $T$ and then go to the limit. Using our hypotheses we get that for some nonnegative $\lambda^{\prime}$

$$
\begin{aligned}
B_{2 k}(t) \leq & B_{2 k}(0)+2 k(C+2 k-1) \mathbb{E} \int_{0}^{t}\left|X_{s}^{i, N}\right|^{2 k-2} d s-2 k \lambda^{\prime} \mathbb{E} \int_{0}^{t}\left|X_{s}^{i, N}\right|^{2 k} d s \\
& -\frac{2 k}{N} \mathbb{E} \sum_{j=1}^{N} \int_{0}^{t}\left(\nabla W\left(X_{s}^{i, N}-X_{s}^{j, N}\right)\right) \cdot X_{s}^{i, N}\left|X_{s}^{i, N}\right|^{2 k-2} d s .
\end{aligned}
$$

To bound the last term above, we use (A3) i.e.

$$
\left|\nabla W\left(X_{s}^{i, N}-X_{s}^{j, N}\right)\right| \leq M\left(1+\left|X_{s}^{i, N}-X_{s}^{j, N}\right|^{m}\right),
$$

and Hölder inequality in order to obtain the following upper bound

$$
2^{(2 k-1) / 2 k} M \int_{0}^{t}\left[\mathbb{E}\left|X_{s}^{i, N}\right|^{2 k}\right]^{\frac{2 k-1}{2 k}}\left[\mathbb{E}\left(1+\left|X_{s}^{i, N}-X_{s}^{j, N}\right|^{2 m k}\right)\right]^{1 / 2 k} d s .
$$

Now we may use exchangeability and Proposition 2.2 to obtain

$$
\begin{aligned}
B_{2 k}(t) \leq & B_{2 k}(0)+2 k(C+2 k-1) \mathbb{E} \int_{0}^{t}\left|X_{s}^{i, N}\right|^{2 k-2} d s-2 k \lambda^{\prime} \mathbb{E} \int_{0}^{t}\left|X_{s}^{i, N}\right|^{2 k} d s \\
& +c(k)\left(1+\mu_{0}\left(|x|^{2 m k}\right)^{1 / 2 k} \int_{0}^{t}\left[\mathbb{E}\left(\left|X_{s}^{i, N}\right|^{2 k}\right)\right]^{\frac{2 k-1}{2 k}} d s .\right.
\end{aligned}
$$

As usual, one can get that $B_{2 k}$ is differentiable and satisfies

$$
B_{2 k}^{\prime}(t) \leq c(k) B_{2 k-2}(t)-2 k B_{2 k}(t)+c(k)\left(1+\mu_{0}\left(|x|^{2 m k}\right)\right) B_{2 k}(t)^{\frac{2 k-1}{k}} .
$$

Since, for every $\varepsilon>0$, it exists $c$ such that $|x|^{2 k-2} \leq c+\varepsilon|x|^{2 k}$ and $a^{\frac{2 k-1}{2 k}} \leq 1+a$ we thus obtain

$$
B_{2 k}^{\prime}(t) \leq c(k)\left(1+\mu_{0}\left(|x|^{2 m k}\right)\right)-\tilde{\lambda} B_{2 k}(t),
$$

for some $\tilde{\lambda}>0$ and we can conclude using Gronwall one more time. $\triangleright$ 
Remark 2.4 If $V$ is identically 0 we may simply remark that

$$
\left|X_{t}^{i, N}\right|^{k} \leq 3^{k-1}\left(\left|X_{0}^{i, N}\right|^{k}+\left|\sqrt{2} B_{t}^{i}\right|^{k}+\left(\frac{1}{N} \sum_{j=1}^{N} \int_{0}^{t} C\left(1+\left|X_{s}^{i, N}-X_{s}^{j, N}\right|^{m}\right) d s\right)^{k}\right)
$$

and directly conclude with the help of Proposition 2.2, thanks to the convexity of $x \rightarrow|x|^{k}$. One could mimic the preceding proof to get the uniform estimate of the moments of the projected particle system under assumptions (A).

\subsection{Identification and existence of solutions of the nonlinear SDE}

We prove here how a tightness criterion may ensure that the empirical law of the particles converges to the solution of the nonlinear SDE (3) proving thus the existence of such a solution.

Lemma 2.5 If $W$ satisfies (A) or $W$ and $V$ satisfy ( $\left.A^{\prime}\right)$, then for all $s$ and $t$ smaller than $T$, all $k \geqslant 2$ and all $i$

$$
\mathbb{E}\left|X_{t}^{i, N}-X_{s}^{i, N}\right|^{2 k} \leq C(k, T)\left(1+\mu_{0}\left(|x|^{m(m+2 k-1)}\right)\right)|t-s|^{\frac{3}{2}} .
$$

\section{Proof}

$\triangleleft$ Let $0 \leq s \leq t \leq T$ and $k \geqslant 1$.

$$
\begin{aligned}
\mathbb{E}\left|X_{t}^{i, N}-X_{s}^{i, N}\right|^{2 k} \leq & 2 k(2 k-1) \mathbb{E} \int_{s}^{t}\left|X_{u}^{i, N}-X_{s}^{i, N}\right|^{2 k-2} d u \\
& +(2 k) \mathbb{E} \int_{s}^{t}\left|\nabla V\left(X_{u}^{i, N}\right)\right|\left|X_{u}^{i, N}-X_{s}^{i, N}\right|^{2 k-1} d u \\
& +\frac{2 k}{N} \mathbb{E} \sum_{j=1}^{N} \int_{s}^{t}\left(\left|\nabla W\left(X_{u}^{i, N}-X_{u}^{j, N}\right)\right|\right)\left|X_{u}^{i, N}-X_{s}^{i, N}\right|^{2 k-1} d u .
\end{aligned}
$$

Hence using (A3), Corollary 2.3 and Hölder (with the best choice of exponents) we get that for $k \geqslant 1$

$$
\mathbb{E}\left|X_{t}^{i, N}-X_{s}^{i, N}\right|^{2 k} \leq C(k, T)\left(1+\mu_{0}\left(|x|^{m(m+2 k-1)}\right)\right)|t-s| .
$$

Plugging this estimate into the previous inequality and using Cauchy-Schwarz, we obtain the desired result. $\triangleright$

It is well known that Lemma 2.5 implies that the sequence of the laws of $\left(s \rightarrow X_{s}^{1, N}\right)_{N}$ defined on $\mathcal{C}\left([0, T], d R^{d}\right)$ is tight. In order to build a solution to the nonlinear SDE (3) we may now follow some standard routine in mean-field particle systems. We here follow the one in [Mél95] Theorem 4.1.4. Thanks to Proposition 4.2.2 in [Mél95] and to Lemma 2.5, the empirical measures $\pi_{N}$ defined on $\mathcal{P}\left(\mathcal{P}\left(\mathcal{C}\left([0, T], \mathbb{R}^{d}\right)\right)\right)$ by $\pi_{N}=1 / N \sum_{i=1}^{N} \delta_{X^{i, N}}$ is tight too. According to the end of section 4.2 in [Mél95], for any limit point $\pi_{\infty}$, any $Q \in \mathcal{P}\left(\mathcal{C}\left([0, T], \mathbb{R}^{d}\right)\right)$ is $\pi_{\infty}$ a.s. a solution to the nonlinear martingale problem (up to time $T$ ). Actually on one hand the proof here is simpler since we have no jumps, but on the other hand the drifts are unbounded (but with polynomial growth) so that to justify passage to the limit we have to use the (uniform in $N$ ) moment estimates in Corollary 2.3 and Proposition 2.2. Details are straightforward and left to the reader, who will check that a sufficient condition is $\mu_{0}\left(|x|^{m^{2}}\right)<+\infty$.

But consider two solutions $(\bar{X}, \bar{Z}$.) of the nonlinear SDE (3), built with the same Brownian motion and the same initial condition, and introduce an independent copy $\left(\bar{X}_{.}^{\prime}, \bar{Z}^{\prime}\right)$. Thanks to Proposition 
2.7 in the next subsection, we know that all processes have a finite second order moment. Hence, it holds

$$
\begin{aligned}
A(t)=\mathbb{E}\left[\left|\bar{X}_{t}-\bar{Z}_{t}\right|^{2}\right] & =-\int_{0}^{t} 2 \mathbb{E}\left[\left(\nabla V\left(\bar{X}_{s}\right)-\nabla V\left(\bar{Z}_{s}\right)\right) \cdot\left(\bar{X}_{s}-\bar{Z}_{s}\right)\right] d s \\
& -\int_{0}^{t} 2 \mathbb{E}\left[\left(\nabla W\left(\bar{X}_{s}-\bar{X}_{s}^{\prime}\right)-\nabla W\left(\bar{Z}_{s}-\bar{Z}_{s}^{\prime}\right)\right) \cdot\left(\bar{X}_{s}-\bar{Z}_{s}\right)\right] d s .
\end{aligned}
$$

But again since $\nabla W(-a)=-\nabla W(a)$ the later integral can be rewritten

$$
-\int_{0}^{t} \mathbb{E}\left[\left(\nabla W\left(\bar{X}_{s}-\bar{X}_{s}^{\prime}\right)-\nabla W\left(\bar{Z}_{s}-\bar{Z}_{s}^{\prime}\right)\right) \cdot\left(\left(\bar{X}_{s}-\bar{X}_{s}^{\prime}\right)-\left(\bar{Z}_{s}-\bar{Z}_{s}^{\prime}\right)\right)\right] d s .
$$

Using (2) and the local Lipschitz property we see that $-(\nabla V(x)-\nabla V(y)) \cdot(x-y) \leq \beta|x-y|^{2}$ a similar result holding for $W$. Using $(a+b)^{2} \leq 2 a^{2}+2 b^{2}$ and the fact that $\bar{X}-\bar{Z}$ and $\bar{X}^{\prime}-\bar{Z}^{\prime}$ have the same law we get

$$
A(t) \leq 3 \beta \int_{0}^{t} A(s) d s
$$

so that $A(t)=0$. Hence we have proved (strong) uniqueness for the nonlinear SDE. As for linear $\mathrm{SDE}$, this notion of uniqueness implies uniqueness in law.

As a byproduct, we obtain that $\pi_{\infty}=\delta_{Q}$ for some $Q$ which is the unique solution of (3) and that $\pi_{N}$ goes to $\delta_{Q}$.

What we have obtained is the following: there exists an unique probability measure $Q$ defined on $C\left([0, T], \mathbb{R}^{d}\right)$ such that for all smooth $f$

$$
(\omega, t) \mapsto f\left(\omega_{t}\right)-f\left(\omega_{0}\right)-\int_{0}^{t}\left(\Delta f\left(\omega_{s}\right)-\nabla V \cdot \nabla f\left(\omega_{s}\right)-\nabla W * Q_{s}\left(\omega_{s}\right) \cdot \nabla f\left(\omega_{s}\right)\right) d s
$$

is a $Q$ martingale, with

$$
\nabla W * Q_{s}\left(\omega_{s}\right)=\int \nabla W\left(\omega_{s}-y\right) Q_{s}(d y)
$$

Let $H(x)=\int \nabla W(x-y) Q_{s}(d y)$. Thanks to Corollary 2.3, $H(x) \leq C\left(1+|x|^{m}\right)$ (provided $\mu_{0}\left(|y|^{m^{2}}\right)$ is finite) and is local Lipschitz (thanks to (A3)). Thus the SDE

$$
d Z_{t}=\sqrt{2} d B_{t}-\nabla V\left(Z_{t}\right) d t-H\left(Z_{t}\right) d t
$$

has a strongly unique solution up to its explosion time. Since $Q$ is a solution, it is the only one. The solution is thus non explosive, and Girsanov theory tells us that $Q$ is absolutely continuous w.r.t. the Wiener measure, provided the drift is of finite energy (see e.g. [CL94] Proposition 2.3), i.e.

$$
\int_{0}^{T} \int\left(|\nabla V|^{2}+|H|^{2}\right)(x) Q_{s}(d x) d s<+\infty .
$$

This condition is satisfied provided $\mu_{0}\left(|x|^{2 m^{2}}\right)<+\infty$. Thus for all $s>0, Q_{s}$ is absolutely continuous w.r.t. Lebesgue measure, with a density $u_{s}$.

Let us summarize our results.

Theorem 2.6 Assume that $W$ satisfies (A) or that $W$ and $V$ satisfy ( $\left.A^{\prime}\right)$. Assume in addition that $\mu_{0}\left(|x|^{a}\right)<+\infty$ for $a=\max \left(m(m+3), 2 m^{2}\right)$.

Then the nonlinear SDE (3) has an unique strong solution $Q$. 
Furthermore for all $t>0, Q_{t}$ (the law of $Q$ at time $t$ ) is absolutely continuous w.r.t. Lebesgue measure, with density $u_{t}$ satisfying for all $T>0$,

$$
\sup _{0<t \leq T} \int|x|^{2 k} u_{t}(x) d x \leq C(T)\left(1+\mu_{0}\left(|x|^{2 m k}\right)\right),
$$

and $t \rightarrow u_{t}$ is a solution of (1).

Finally $t \rightarrow u_{t} d x$ is the unique solution of (1) among the set of continuous flows of measures $t \rightarrow \nu_{t}$ satisfying for all $T>0$,

$$
\int_{0}^{T} \int|x|^{2 m^{2}} d \nu_{t} d t<+\infty
$$

The only thing it remains to prove is the last statement. Let $t \rightarrow \nu_{t}$ be a solution of (1) satisfying the (finite energy) condition above. Then, according to Theorem 4.18 in [CL94], a solution of the (linear time inhomogeneous) SDE

$$
d Z_{t}=\sqrt{2} d B_{t}-\nabla V\left(Z_{t}\right) d t-\left(\nabla W * \nu_{t}\right)\left(Z_{t}\right) d t
$$

with initial law $\mu_{0}$ exists and furthermore its law at time $t$ is given by $\nu_{t}$. Hence $Z$. is a solution of the nonlinear SDE. Uniqueness of the later implies that $\nu_{t}=u_{t} d x$.

About the literature. If existence and uniqueness have been extensively discussed in the framework of mean field interacting particle systems with bounded interactions, the case of unbounded interactions was not much studied. The above proof shows that convexity at infinity allows us to essentially mimic the bounded case, just changing the tightness criterion to be used, and using some recent aspects of stochastic calculus related to singular diffusion processes.

If $d=1$ another version of Theorem 2.6 is obtained in [BRTV98] Theorem 3.1 following a completely different way. One can notice that some moment condition on $\mu_{0}$ similar to ours is also required therein. Our condition (as well as the one in [BRTV98]) is certainly non sharp. If a large part of the method in [BRTV98] can be extended to the $d$ dimensional case, some aspects (strongly using monotonicity) require additional work.

For the nonlinear PDE, for $d=1$ and $m=2$ an existence and uniqueness result is stated in [BCCP98] p.983. However the initial measure has to be absolutely continuous with a $\mathcal{C}^{2}$ density satisfying $\int|x|^{4} d \mu_{0}<+\infty$ (while we need a 10 instead of a 4 ), and uniqueness holds for classical $\left(\mathcal{C}^{2}\right)$ solutions.

The conclusion is that such results are certainly not useless. Since we shall need stronger moment assumptions in the sequel, we did not try to obtain the sharpest conditions in Theorem 2.6 (the interested reader may indeed remark that one need for the use of lemma 2.5 for tension only a dependence in time of the order $|t-s|^{1+\epsilon}$ so that the condition on the initial measure may be weakened).

\subsection{Uniform moment control for the nonlinear SDE}

We start with the proof of the moment control for any solution of the nonlinear SDE.

Proposition 2.7 Assume that $W$ satisfies ( $A$ ) or that $W$ and $V$ satisfy ( $A$ '). Then

$$
\sup _{t \geqslant 0} \mathbb{E}\left|\bar{X}_{t}\right|^{2} \leq \mu_{0}\left(|x|^{2}\right)+K \text {. }
$$

Proof

$\triangleleft$ Let $s \rightarrow \bar{X}_{s}^{\prime}$ an independent copy of $s \rightarrow \bar{X}_{s}$. Then

$$
\begin{aligned}
\mathbb{E}\left(\left|\bar{X}_{t}\right|^{2}\right) & =\mathbb{E}\left(\left|\bar{X}_{0}\right|^{2}\right)-2 k \int_{0}^{t} \mathbb{E}\left(\bar{X}_{s} \cdot\left(\nabla V\left(\bar{X}_{s}\right)+\nabla W * u_{s}\left(\bar{X}_{s}\right)\right)\right) d s+2 d t \\
& =\mathbb{E}\left(\left|\bar{X}_{0}\right|^{2}\right)-2 \int_{0}^{t} \mathbb{E}\left(\bar{X}_{s} \cdot \nabla V\left(\bar{X}_{s}\right)\right) d s-\int_{0}^{t} \mathbb{E}\left(\left(\bar{X}_{s}-\bar{X}_{s}^{\prime}\right) \cdot \nabla W\left(\bar{X}_{s}-\bar{X}_{s}^{\prime}\right)\right) d s+2 d t
\end{aligned}
$$


so that if we denote $v(t)=\mathbb{E}\left(\left|\bar{X}_{t}\right|^{2}\right)$, we may first differentiate w.r.t. time as we did before, and then use the hypotheses. Remark that, since $W$ is convex at infinity and symmetric,

$$
x \cdot \nabla W(x) \geq x \cdot \nabla W(0)+\lambda_{W}|x|^{2}-C \geqslant-C .
$$

It follows

$$
v^{\prime}(t) \leq-2 \lambda_{V} v(t)+(2 d+3 C)-2 \lambda_{W}\left(v(t)-\mathbb{E}^{2}\left(\bar{X}_{t}\right)\right) .
$$

If (A') holds, $\lambda_{V}>0$ and Gronwall's lemma concludes the proof. If $V=0, \lambda_{W}>0$ and we have assumed that $E\left(\bar{X}_{t}\right)=0$ so that Gronwall's lemma concludes the proof. $\triangleright$

Now, if we assume that existence and uniqueness hold for the non linear SDE, the results in the previous two subsections imply some control of the moment of the solution, just taking limits. But the previous a priori bound was necessary to complete the proof of Theorem 2.6.

\section{Propagation of chaos}

This section is devoted to the comparison of the behavior, for a fixed number of particles, of the difference between one particle and the solution of the non-linear SDE. Ideally, it will be uniform on time and will decrease quickly to 0 as the number of particles increases. We will however see that to get such an estimate, we have to introduce a new convexity assumption, ensuring strict convexity except at some points.

All results in this section are written under assumption (A) i.e. with a vanishing confinement potential $V$. Replacing $Y$ by $X$, the same results hold under assumption (A'), modifying the proofs in the same way as we did for various statements in the previous section.

\subsection{A first control on the error}

We prove here a first uniform control on the mean square error between one particle and the solution of the nonlinear SDE. In the sequel we shall always make assumptions ensuring existence and uniqueness of strong solutions. Hence we may build solutions for (8) (or (7)) and (3) with the same Brownian motions and the same initial random variables (obtaining thus and i.i.d. sample $\left(\bar{X}_{.}^{i}\right)_{i=1, \ldots, N}$ of $\left.\bar{X}_{.}\right)$.

Theorem 3.1 Assume that $W$ satisfies (A). Suppose that the law $\mu_{0}$ admits a large enough polynomial moment. Then there exists $K>0$ such that

$$
\sup _{t \geq 0} \mathbb{E}\left(\left|Y_{t}^{i, N}-\bar{X}_{t}^{i}\right|^{2}\right) \leq K
$$

This result is of course not so good as it doesn't imply the so called uniform propagation of chaos, since the last bound does not tend to 0 as $N$ tends to infinity. However under the condition that $W$ is the sum of an uniformly convex function and a Lipschitz compactly supported one, one may show the following bound, for some positive $a$

$$
\mathbb{E}\left(\left|Y_{t}^{i, N}-\bar{X}_{t}^{i}\right|^{2}\right) \leq K e^{a t} / N
$$

which gives us the non uniform propagation of chaos. The proof of such an estimate is the conjunction of the proof below and standard estimates for bounded Lipschitz drifts for mean field particle systems (see again [Mél95]).

Proof

$\triangleleft$ Introduce

$$
\bar{Y}_{t}^{i, N}=\bar{X}_{t}^{i}-\frac{1}{N} \sum_{j=1}^{N} \bar{X}_{t}^{j}
$$


Then

$$
\begin{aligned}
\mathbb{E}\left(\left|Y_{t}^{i, N}-\bar{X}_{t}^{i}\right|^{2}\right) & \leq 2 \mathbb{E}\left(\left|Y_{t}^{i, N}-\bar{Y}_{t}^{i}\right|^{2}\right)+2 \mathbb{E}\left(\left|\bar{Y}_{t}^{i, N}-\bar{X}_{t}^{i}\right|^{2}\right) \\
& =2 \mathbb{E}\left(\left|Y_{t}^{i, N}-\bar{Y}_{t}^{i}\right|^{2}\right)+\frac{2}{N} \mathbb{E}\left(\left|\bar{X}_{t}^{i}\right|^{2}\right)
\end{aligned}
$$

since $\mathbb{E}\left(\bar{X}_{t}^{i}\right)=0$ and the $\bar{X}_{t}^{j}$ s are independent. The second term is bounded by some $K / N$ according to Proposition 2.7 so that we focus on the first term. But

$$
\begin{aligned}
Y_{t}^{i, N}-\bar{Y}_{t}^{i, N}= & -\frac{1}{N} \int_{0}^{t} \sum_{j=1}^{N}\left[\nabla W\left(Y_{s}^{i, N}-Y_{s}^{j, N}\right)-\nabla W * u_{s}\left(\bar{X}_{s}^{i}\right)\right] d s \\
& -\frac{1}{N} \int_{0}^{t} \sum_{j=1}^{N} \nabla W * u_{s}\left(\bar{X}_{s}^{j}\right) d s
\end{aligned}
$$

so that

$$
\sum_{i=1}^{N}\left|Y_{t}^{i, N}-\bar{Y}_{t}^{i, N}\right|^{2}=-\frac{2}{N} \sum_{i, j=1}^{N} \int_{0}^{t}\left(A_{i j}(s)+B_{i j}(s)+C_{i j}(s)\right) d s
$$

with

$$
\begin{aligned}
& A_{i j}(s)=\left(\nabla W\left(Y_{s}^{i, N}-Y_{s}^{j, N}\right)-\nabla W\left(\bar{Y}_{s}^{i, N}-\bar{Y}_{s}^{j, N}\right)\right) \cdot\left(Y_{s}^{i, N}-\bar{Y}_{s}^{i, N}\right), \\
& B_{i j}(s)=\left(\nabla W\left(\bar{X}_{s}^{i}-\bar{X}_{s}^{j}\right)-\nabla W * u_{s}\left(\bar{X}_{s}^{i}\right)\right) \cdot\left(Y_{s}^{i, N}-\bar{Y}_{s}^{i, N}\right), \\
& \left.C_{i j}(s)=\nabla W * u_{s}\left(\bar{X}_{s}^{i}\right)\right) \cdot\left(Y_{s}^{i, N}-\bar{Y}_{s}^{i, N}\right) .
\end{aligned}
$$

Let us deal with the first term,

$$
\begin{aligned}
& \sum_{i, j=1}^{N} A_{i j}(s)=\frac{1}{2} \sum_{i, j=1}^{N}\left(A_{i j}(s)+A_{j i}(s)\right) \\
& \quad=\frac{1}{2} \sum_{i, j=1}^{N}\left(\nabla W\left(Y_{s}^{i, N}-Y_{s}^{j, N}\right)-\nabla W\left(\bar{Y}_{s}^{i, N}-\bar{Y}_{s}^{j, N}\right)\right) \cdot\left(\left(Y_{s}^{i, N}-Y_{s}^{j, N}\right)-\left(\bar{Y}_{s}^{i, N}-\bar{Y}_{s}^{j, N}\right)\right) \\
& \quad \geq \lambda N \sum_{i=1}^{N}\left|Y_{s}^{i, N}-\bar{Y}_{s}^{i, N}\right|^{2}-C N^{2} / 2
\end{aligned}
$$

using (2) and properties of vectors in $\mathcal{M}$.

Remark now that

$$
\begin{aligned}
-\mathbb{E} \sum_{j=1}^{N} B_{i j}(s) & \leq\left(\mathbb{E}\left|Y_{s}^{i, N}-\bar{Y}_{s}^{i, N}\right|^{2}\right)^{1 / 2}\left(\mathbb{E} \mid \sum_{j=1}^{N}\left(\nabla W\left(\bar{X}_{s}^{i}-\bar{X}_{s}^{j}\right)-\left.\nabla W * u_{s}\left(\bar{X}_{s}^{i}\right)\right|^{2}\right)^{1 / 2}\right. \\
& \leq c \sqrt{N}\left(\mathbb{E}\left|Y_{s}^{i, N}-\bar{Y}_{s}^{i, N}\right|^{2}\right)^{1 / 2}
\end{aligned}
$$

by Cauchy-Schwarz inequality, the polynomial growth of $\nabla W$, the controls of moments previously established and the key remark

$$
\mathbb{E}\left(\nabla W\left(\bar{X}_{s}^{i}-\bar{X}_{s}^{j}\right)-\nabla W * u_{s}\left(\bar{X}_{s}^{i}\right)\right)=0
$$

The last term involving $C_{i j}(s)$ is controlled using the same tools. We finally get, defining

$$
\alpha(s)=\mathbb{E}\left(\left|Y_{s}^{i, N}-\bar{Y}_{s}^{i, N}\right|^{2}\right)
$$


and reasoning as we did in the previous section, the differential inequality

$$
\alpha^{\prime}(s) \leq-2 \lambda \alpha(s)+\frac{c}{\sqrt{N}} \sqrt{\alpha(s)}+C / 2 .
$$

Now we may use $\sqrt{\alpha(s)} / \sqrt{N} \leq 1 / 2\left(\varepsilon^{-1} \frac{1}{N}+\varepsilon \alpha(s)\right)$ and choose $\varepsilon=2 \lambda / c$. The previous inequality becomes a classical

$$
\alpha^{\prime}(s) \leq-\lambda \alpha(s)+(c / \varepsilon N)+C / 2
$$

for which we can use Gronwall's lemma. $\triangleright$

Remark. When $C=0$ we get the uniform (in time) propagation of chaos result obtained in [Mal03] with rate $1 / N$. Of course in our case (convexity at infinity only) such an uniform propagation of chaos cannot be expected (more precisely cannot be shown with usual tools). Indeed, the collision potential $W$ obliges the particle to mainly stay in the same region, where there is no more attraction between particles.

\subsection{Uniform propagation of chaos}

In view of Theorem 3.1 and its proof, we have to reinforce the convexity assumption in order to prove the uniform propagation of chaos phenomenon.

The condition (6) in the introduction and inspired by the work of Carrillo-McCann-Villani [CMCV06] is recalled below:

we say that condition $\mathbf{C}(\mathbf{A}, \alpha)$ holds if there exist $A, \alpha>0$ such that for any $0<\epsilon<1$,

$$
\forall x, y \in \mathbb{R}^{d}, \quad(x-y) \cdot(\nabla W(x)-\nabla W(y)) \geq A \epsilon^{\alpha}\left(|x-y|^{2}-\epsilon^{2}\right) .
$$

We can now prove

Theorem 3.2 Assume that $W$ satisfies $\mathbf{C}(\mathbf{A}, \alpha)$ and $(A)$. Suppose that the law $\mu_{0}$ has a large enough polynomial moment. Then there exists $K>0$ such that

$$
\sup _{t \geq 0} \mathbb{E}\left(\left|Y_{t}^{i, N}-\bar{X}_{t}^{i}\right|^{2}\right) \leq \frac{K}{N^{\frac{1}{1+\alpha}}}
$$

\section{Proof}

$\triangleleft$ The proof follows the same lines than the one of Theorem 3.1. First recall that

$$
\mathbb{E}\left(\left|Y_{t}^{i, N}-\bar{X}_{t}^{i}\right|^{2}\right) \leq 2 \mathbb{E}\left(\left|Y_{t}^{i, N}-\bar{Y}_{t}^{i}\right|^{2}\right)+\frac{2}{N} \mathbb{E}\left(\left|\bar{X}_{t}^{i}\right|^{2}\right)
$$

and the second term is of a better order thanks to Proposition 2.7 so that we focus on the first term. For this term the only modification is the control of $A_{i j}(s)$ where we replace convexity at infinity by (6). This yields

$$
\sum_{i, j=1}^{N} A_{i j}(s) \geq A \epsilon^{\alpha}\left(N \sum_{i=1}^{N}\left|Y_{s}^{i, N}-\bar{Y}_{s}^{i, N}\right|^{2}-\epsilon^{2} N^{2} / 2\right),
$$

so that the differential inequality satisfied by $\alpha(t)$ becomes

$$
\alpha^{\prime}(s) \leq-2 A \epsilon^{\alpha}\left(\alpha(s)-\epsilon^{2}\right)+\frac{c}{\sqrt{N}} \sqrt{\alpha(s)} .
$$

Applying Theorem 3.1 we know that $\alpha(s) \leq K$ for some $K>1$.

We may take $\epsilon=\sqrt{\alpha(s)} / 2 \sqrt{K}<1$ and get

$$
\alpha^{\prime}(s) \leq-J \alpha(s)^{1+\alpha / 2}+\frac{c}{\sqrt{N}} \sqrt{\alpha(s)}
$$


with $J=\frac{2 A}{(2 \sqrt{K})^{\alpha}}\left(1-\frac{1}{4 K}\right)$. Define $\beta(s)=\sqrt{\alpha(s)}$. Then

$$
\beta^{\prime}(s)+(J / 2) \beta^{1+\alpha}(s) \leq \frac{c}{2 \sqrt{N}}
$$

so that

$$
\beta(s) \leq C / N^{1 / 2(1+\alpha)}
$$

for any $s$ such that $\beta^{\prime}(s) \geq 0$. Since $\beta(0)=0$ it easily follows that $\beta(s) \leq C / N^{1 / 2(1+\alpha)}$ everywhere, hence the result. $\triangleright$

Remark. If (A') holds we have to assume that $V$ satisfies $\mathbf{C}(\mathbf{A}, \alpha)$ and $W$ is convex.

\section{Ergodicity of the nonlinear SDE}

\subsection{Drift condition and existence of a stationary measure}

In fact, as long as we consider only the particle system, the condition (2) is sufficient to ensure the ergodicity, and even exponential ergodicity, of the particle system. Indeed we have that $\left(X_{t}^{i, N}\right)_{1 \leq i \leq N}$ is strongly Feller aperiodic and we can use Down-Meyn-Tweedie's drift condition [DMT95]. This condition is the following: there exists some $\Phi \geq 1$ with compact level sets such that for some positive $\lambda, b$

$$
\mathcal{L} \Phi \leq-\lambda \Phi+b
$$

where $\mathcal{L}$ is the generator of the particle system. It is trivially verified here with the function $\Phi(x)=\|x\|^{2}+1$ according to (2). It of course implies the existence of an invariant measure $\pi^{N}$ and that there exists $\delta(N)>0$ such that denoting $P_{t}^{N}$ the semigroup associated with the particle system

$$
\left\|P_{t}^{N}(x, \cdot)-\pi^{N}(\cdot)\right\|_{T V} \leq c e^{-t \delta(N)} \Phi(x) .
$$

As a byproduct, we get the existence of an invariant measure for the nonlinear SDE (hence an equilibrium for the nonlinear PDE), provided we have uniform (in time) propagation of chaos. Indeed the previous inequality together with uniform propagation of chaos show that the family $\left(u_{t}\right)_{t>0}$ is a Cauchy family in $\mathbf{L}^{1}(d x)$, which is a complete space.

Such a result is however not useful to control the convergence to equilibrium for the nonlinear SDE (or even the nonlinear PDE). Indeed there is no close form for $\delta(N)$ which heavily depends on $N$, so that even the uniform propagation of chaos cannot give good estimations for the rate of convergence of $\left(\bar{X}_{t}\right)$ to its invariant measure. Note that even a strict convexity condition does not seem to be useful for such an approach.

\subsection{Logarithmic Sobolev inequality}

Under a global uniform convexity condition (i.e. $C=0$ in (2)), Malrieu proves in [Mal03] that the invariant measure of the centered particles system i.e.

$$
u_{\infty}^{N}(y) d y=Z_{N}^{-1} \exp \left(-\frac{1}{2 N} \sum_{i, j=1}^{N} W\left(y_{i}-y_{j}\right)\right) d y
$$

where $d y$ is Lebesgue measure on the hyperplane $\mathcal{M}$, satisfies a logarithmic Sobolev inequality with a constant $1 / \lambda$ independent of the dimension using the famous Bakry-Émery criterion. So that if $u_{t}^{N}$ stands for the law of $\left(Y_{t}^{i, N}\right)_{1 \leq i \leq N}$ we have that

$$
\operatorname{Ent}\left(u_{t}^{N} \mid u_{\infty}^{N}\right) \leq e^{-2 \lambda t} \operatorname{Ent}\left(u_{0}^{N} \mid u_{\infty}^{N}\right) .
$$


Projecting on the first coordinate, using a $T_{2}$ inequality and, on one hand the dimension free exponential convergence, and on the other hand the uniform (in time) propagation of chaos, he essentially proves the following

$$
W_{2}\left(u_{t}, u_{\infty}\right) \leq C e^{-\lambda t} \sqrt{\operatorname{Ent}\left(u_{0} \mid u_{\infty}\right)} .
$$

This result was obtained by Carrillo-McCann-Villani [CMCV03], and improved in Carrillo-McCannVillani [CMCV06] where the authors replace the square root of the initial entropy by $W_{2}\left(u_{0}, u_{\infty}\right)$ (recall that a log-Sobolev inequality implies a $T_{2}$ inequality, i.e. $\left.W_{2}\left(u_{0}, u_{\infty}\right) \leq C \sqrt{\operatorname{Ent}\left(u_{0} \mid u_{\infty}\right)}\right)$.

It is of course tempting to use the same approach in the non strictly convex case.

The first point is that under assumption $\mathbf{C}(\mathbf{A}, \alpha)$, we may no longer use Bakry-Émery condition for the measure $u_{\infty}^{N}$ as the Hessian may degenerate. We are thus obliged to use some perturbation argument.

To fix ideas and to simplify the presentation, let us restrict ourselves to a polynomial potential, namely $W(x)=|x|^{2+\alpha}$. Consider first a crude attempt: let $\beta(x)=a x^{2} /\left(1+x^{2}\right)$ with some positive $a$ : $\beta$ is bounded, and has positive curvature $2 a$ at 0 . Choose now $a=1 / N$ and consider the perturbed measure

$$
\nu_{\infty}^{N}(d y)=Z_{N}^{\prime} \exp \left(\frac{1}{N} \sum_{i, j} \beta\left(\left|y_{i}-y_{j}\right|\right)\right) u_{\infty}^{N}(d y) .
$$

By arguments developed in Malrieu [Mal03] one may then easily establish that $\nu_{\infty}^{N}$ has positive curvature bounded below by $1 / N$. Thus it satisfies a logarithmic Sobolev inequality with constant $e N$ remarking that the perturbation potential has oscillations bounded by one and applying the standard Holley-Stroock perturbation argument. We may then use the entropic convergence associated to such a logarithmic Sobolev inequality, the $T_{2}$ inequality projected on the first coordinate and uniform propagation of chaos (Theorem 3.2) to get

$$
W_{2}\left(u_{t}, u_{\infty}\right) \leq\left(\frac{K}{N^{\frac{1}{1+\alpha}}}\right)^{1 / 2}+e^{-t / 4 e N} \sqrt{2 e N \operatorname{Ent}\left(u_{0} \mid u_{\infty}\right)}
$$

Optimizing in $N$ ensures that

$$
W_{2}\left(u_{t}, u_{\infty}\right) \leq \frac{K^{\prime} \log ^{\frac{1}{2(1+\alpha)}} t}{t^{\frac{1}{2(1+\alpha)}}}
$$

which is quite a bad estimate of the speed since Carrillo-McCann-Villani obtained in such a case a speed $t^{-(1 / \alpha)}$ (we shall recover this speed in the next subsection).

Note finally that this approach via logarithmic Sobolev inequality has two major drawbacks: on one hand the right hand side depends on the entropy of the initial data, on the other hand it cannot be used to compare the behavior of two solutions starting from different initial measures. We will see in the next section how to solve both these problems by a direct simple approach.

\subsection{A direct control of the $\mathrm{L}^{2}$-Wasserstein distance}

The goal of this section is to show that even if the logarithmic Sobolev inequality cannot give useful result, one may use a direct approach to obtain a (uniform in $N$ ) control of the Wasserstein distance between two solutions of the particle system starting from different points. So, combining this with the uniform in time propagation of chaos, we recover in an elegant and simple way the results of Carrillo-McCann-Villani [CMCV06].

Theorem 4.1 Assume that $W$ satisfies $(A)$ and the convexity condition $\mathbf{C}(A, \alpha)$. Let $u_{t}$ and $v_{t}$ be the unique solutions of the nonlinear PDE with initial conditions respectively $u_{0}$ and $v_{0}$. We assume for simplicity that both $u_{0}$ and $v_{0}$ have an exponential moment (or a large enough polynomial moment in order to ensure existence and uniqueness). 
Then $t \mapsto W_{2}^{2}\left(u_{t}, v_{t}\right)$ is non-increasing. Furthermore there exists $t_{1} \leq\left(2^{2+\alpha} / 3\right) \log \left(W_{2}^{2}\left(u_{0}, v_{0}\right)\right) / A$ such that

$$
\begin{aligned}
W_{2}^{2}\left(u_{t}, v_{t}\right) & \leq e^{-\left(3 A / 2^{2+\alpha}\right) t} W_{2}^{2}\left(u_{0}, v_{0}\right) \quad \text { if } t<t_{1}, \\
& \leq\left(1+A(\alpha /(2+\alpha))^{1+\alpha / 2}\left(t-t_{1}\right)\right)^{-2 / \alpha} \quad \text { if } t \geq t_{1} .
\end{aligned}
$$

In particular, if $t>(1+\eta)\left(2^{2+\alpha} / 3\right) \log \left(W_{2}^{2}\left(u_{0}, v_{0}\right)\right) / A$ for some $\eta>0$, one has

$$
W_{2}^{2}\left(u_{t}, v_{t}\right) \leq\left(1+(A /(1+\eta))(\alpha /(2+\alpha))^{1+\alpha / 2} t\right)^{-2 / \alpha} .
$$

In addition for all $t \geq 0$ one has

$$
W_{2}^{2}\left(u_{t}, v_{t}\right) \leq\left(W_{2}^{-\alpha}\left(u_{0}, v_{0}\right)+A(\alpha /(2+\alpha))^{1+\alpha / 2} t\right)^{-2 / \alpha} .
$$

Under an uniform convexity condition for $W$ (i.e. $\mathbf{C}(A, 0)$ ), the convergence is exponential i.e.

$$
W_{2}\left(u_{t}, v_{t}\right) \leq C e^{-A t} W_{2}\left(u_{0}, v_{0}\right) .
$$

Note that the final assertion completes the result of Malrieu [Mal03] who cannot compare via logarithmic Sobolev inequalities the distance between two solutions.

Remark also that the bound obtained for the control in Wasserstein distance in the convergence towards the stationary measure is also better as it is now $W_{2}\left(u_{0}, u_{\infty}\right)$ which controls the decay and which is smaller (since a $T_{2}$ inequality holds) than $C \sqrt{\operatorname{Ent}\left(u_{0} \mid u_{\infty}\right)}$.

This will be explained later.

As will be seen from the proof, there is no use here of heavy (but sharp) technology as optimal transport in length space as developed by Carrillo-McCann-Villani [CMCV06] and thus we believe that such an approach will give good results for other models (reinforced diffusion,...).

Proof

$\triangleleft$ In what follows we shall always consider coupling consisting in picking the same Brownian motions $\left(B^{i}\right)$ for the two particle systems, and only choose an ad-hoc coupling for the initial random variables. Denote $u_{t}^{1, N}$ the law of the first particle of the centered particle system $(Y)$ starting with initial law $u_{0}$ and consider $v_{t}^{1, N}$ the law of the first particle of the centered particle system starting with initial law $v_{0}$. Let us first remark that for each coupling $g_{0}$ of $u_{0}$ and $v_{0}$

$$
W_{2}^{2}\left(u_{t}^{1, N}, v_{t}^{1, N}\right) \leq \frac{1}{N} \mathbb{E}_{g_{0}}\left(\sum_{i=1}^{N}\left|Y_{t}^{i, N}-Y_{t}^{i, N}\right|^{2}\right)
$$

where $\left(Y^{i, N}\right)$ is given by the centered particle system where each particle starts with law $u_{0},\left(Y^{\prime i, N}\right)$ with measure $v_{0}$ and the initial law of the pairs $\left(Y_{0}^{i, N}, Y_{0}^{i, N}\right)$ are given by independent copies of $g_{0}$. The subscript for the expectation is related to the initial law.

We then get by Itô's formula and symmetry (starting now from points $y$ and $y^{\prime}$ )

$$
\begin{aligned}
\mathbb{E}_{y, y^{\prime}} & \left(\sum_{i=1}^{N}\left|Y_{t}^{i, N}-Y_{t}^{i, N}\right|^{2}\right) \\
= & \sum_{i=1}^{N}\left|y_{i}-y_{i}^{\prime}\right|^{2}-\frac{1}{N} \sum_{i, j} \mathbb{E}_{y, y^{\prime}} \int_{0}^{t}\left\langle Y_{s}^{i, N}-Y_{s}^{\prime i, N}, \nabla W\left(Y_{s}^{i, N}-Y_{s}^{j, N}\right)-\nabla W\left(Y_{s}^{\prime i, N}-Y_{s}^{\prime j, N}\right)\right\rangle d s \\
= & \sum_{i=1}^{N}\left|y_{i}-y_{i}^{\prime}\right|^{2}-\frac{1}{2 N} \sum_{i, j} \mathbb{E}_{y, y^{\prime}} \int_{0}^{t} \xi_{i j}(s) d s,
\end{aligned}
$$


with

$$
\xi_{i j}(s)=\left\langle\left(Y_{s}^{i, N}-Y_{s}^{j, N}\right)-\left(Y_{s}^{i, N}-Y_{s}^{\prime j, N}\right), \nabla W\left(Y_{s}^{i, N}-Y_{s}^{j, N}\right)-\nabla W\left(Y_{s}^{i, N}-Y_{s}^{\prime j, N}\right)\right\rangle .
$$

We may now differentiate in time and then use condition $\mathbf{C}(A, \alpha)$ to get

$$
\begin{aligned}
\frac{d}{d t} \mathbb{E}_{y, y^{\prime}}\left(\sum_{i=1}^{N}\left|Y_{t}^{i, N}-Y_{t}^{i, N}\right|^{2}\right) & =-\frac{A \epsilon^{\alpha}}{2 N} \sum_{i, j} \mathbb{E}_{y, y^{\prime}}\left(\left|\left(Y_{t}^{i, N}-Y_{t}^{j, N}\right)-\left(Y_{t}^{i, N}-Y_{t}^{\prime j, N}\right)\right|^{2}-\epsilon^{2}\right) \\
& =-A \epsilon^{\alpha} \mathbb{E}_{y, y^{\prime}} \sum_{i}\left(\left|Y_{t}^{i, N}-Y_{t}^{\prime i, N}\right|^{2}-\epsilon^{2}\right)
\end{aligned}
$$

which gives us by exchangeability

$$
\frac{d}{d t} \mathbb{E}_{y, y^{\prime}}\left(\left|Y_{t}^{i, N}-Y_{t}^{i, N}\right|^{2}\right) \leq-A \epsilon^{\alpha} \mathbb{E}\left(\left|Y_{t}^{i, N}-Y_{t}^{\prime i, N}\right|^{2}-\epsilon^{2}\right)
$$

Denote $\xi(t)=\mathbb{E}_{y, y^{\prime}}\left(\left|Y_{t}^{i, N}-Y_{t}^{i, N}\right|^{2}\right)$, we then obtain for all $\epsilon<1$

$$
\xi^{\prime}(t) \leq-A \epsilon^{\alpha}\left(\xi(t)-\epsilon^{2}\right)
$$

This inequality for $\varepsilon$ going to 0 , implies $\xi^{\prime}(t) \leq 0$, i.e. $\xi$ is non-increasing. As a byproduct we get that $t \mapsto W_{2}\left(u_{t}^{1, N}, v_{t}^{1, N}\right)$ is also non-increasing. Indeed if we choose $g_{0}$ as the optimal coupling for the quadratic cost, it holds

$$
W_{2}^{2}\left(u_{0}, v_{0}\right)=\xi(0) \geq \xi(t) \geq W_{2}^{2}\left(u_{t}^{1, N}, v_{t}^{1, N}\right),
$$

and we get the result just shifting the initial time.

Now we can separate two cases: either $\xi(t)>1$ or $\xi(t) \leq 1$. Note that there exists some $t_{1} \geq 0$ such that the first case holds for $t<t_{1}$ and the second one for $t \geq t_{1}$.

If $t<t_{1}$ we may choose any $\varepsilon$, for instance here we choose $\varepsilon=1 / 2$ and obtain (since $\xi(t)>1$, $\left.\varepsilon^{2}=1 / 4<\xi(t) / 4\right)$

$$
\xi^{\prime}(t) \leq-A(\alpha) \xi(t)
$$

with $A(\alpha)=(3 A / 4)(1 / 2)^{\alpha}$, which gives by Gronwall's lemma

$$
\xi(t) \leq e^{-A(\alpha) t} \xi(0) .
$$

Choosing again the optimal coupling $g_{0}$ we obtain the first part of the result and the fact that $t_{1} \leq \log \left(W_{2}^{2}\left(u_{0}, v_{0}\right)\right) / A(\alpha)$.

For the second part, if $t \geq t_{1}$ choose $\epsilon^{2}=\alpha \xi(t) /(\alpha+2)$ to get

$$
\xi^{\prime}(t) \leq-A\left(\frac{\alpha}{2+\alpha}\right)^{\alpha / 2} \frac{2}{2+\alpha} \xi(t)^{1+\alpha / 2} .
$$

Integrating this differential inequality we get

$$
\xi(t) \leq\left(1+B(\alpha)\left(t-t_{1}\right)\right)^{-2 / \alpha},
$$

with $B(\alpha)=A(\alpha /(2+\alpha))^{1+\alpha / 2}$. Our choice of $\varepsilon$ gives the optimal constant (for this method). Of course writing $\mathbf{C}(A, \alpha)$ as we did is a little bit artificial, and if we want a more homogeneous estimate we may remark that $\mathbf{C}(A, \alpha)$ implies

$$
(x-y) \cdot(\nabla W(x)-\nabla W(y)) \geq A\left(\epsilon / W_{2}\left(u_{0}, v_{0}\right)\right)^{\alpha}\left(|x-y|^{2}-\epsilon^{2}\right)
$$


for $\epsilon<W_{2}\left(u_{0}, v_{0}\right)$. So we may always choose $\epsilon^{2}=\alpha \xi(t) /(\alpha+2)$ and get for all $t$,

$$
\xi(t) \leq\left(W_{2}^{-\alpha}\left(u_{0}, v_{0}\right)+B(\alpha) t\right)^{-2 / \alpha} .
$$

We use then the uniform propagation of chaos property to transfer the inequality from the particles to the solutions of the nonlinear SDE:

$$
W_{2}\left(u_{t}, v_{t}\right) \leq W_{2}\left(u_{t}, u_{t}^{1, N}\right)+W_{2}\left(u_{t}^{1, N}, v_{t}^{1, N}\right)+W_{2}\left(v_{t}, v_{t}^{1, N}\right),
$$

and take the previous (uniform in $N$ ) estimation for the middle term and the uniform in time estimation for the first and third term and let $N$ go to infinity. Of course if $t_{1}$ depends on $N$, its bound does not, so that we may find a converging subsequence and get the result. $\quad \triangleright$

Remarks. We have seen in subsection 4.1 that there exists an invariant measure $u_{\infty}$ for the nonlinear PDE. The bound for $W_{2}\left(u_{t}, v_{t}\right)$ obtained by introducing the particle system of order $N$, and then choosing choosing $v_{0}=u_{\infty}^{N}$ allows us to prove this existence too (using completeness of the Wasserstein distance). In addition the decay of the Wasserstein distance trivially implies uniqueness of $u_{\infty}$, at least in the set of measures having some large enough polynomial moment.

Hence, we recover in the previous result the asymptotic rate of convergence to equilibrium obtained by Carrillo-McCann-Villani. But it seems that the result improves upon theirs for small times where we are able to describe some exponential decay. However the value of $t_{1}$ is not explicit, so that for practical issues, this initial exponential decay is not really tractable.

We choose here to develop the point of view of the particles; prove the decay of the Wasserstein distance for the particles system and then transfer this decay to the solution of the nonlinear SDE via uniform propagation of chaos. It is however easy to develop the same line of proof directly for the control of the Wasserstein distance of two solutions of the nonlinear SDE.

Note finally that we did not consider here coefficient diffusion other than constant, as granular media equation is formulated with a constant one. It would however not be difficult to introduce a condition enabling to obtain the same decay. Indeed consider

$$
d Z_{t}=b\left(Z_{t}\right) d t+\sigma\left(Z_{t}\right) d W_{t}
$$

where $W_{t}$ is the usual Wiener process in $\mathbb{R}^{d}$, and assume that for all $0<\epsilon<1$ there exists positive $A$ and $\alpha$ such that

$$
(x-y) \cdot(b(x)-b(y))+\frac{1}{2} \operatorname{tr}\left((\sigma(x)-\sigma(y))(\sigma(x)-\sigma(y))^{t}\right) \leq-A \epsilon^{\alpha}\left(\|x-y\|^{2}-\epsilon^{2}\right)
$$

then we easily derive the same polynomial decay for the Wasserstein distance of two solutions $Z_{t}$ and $\tilde{Z}_{t}$ with different initial conditions. Note that this type of condition hold with possible degeneracy of the diffusion coefficient.

Again in this section we should assume that (A') holds, $V$ satisfies $\mathbf{C}(\mathbf{A}, \alpha)$ and $W$ is convex.

\section{Concentration inequality}

The main goal of this section is to complete the results on convergence of the particle system and of the nonlinear system by providing a (non asymptotic) deviation inequality. This inequality will be written for additive functionals of the particles and then allows us to estimate integrals with respect to the stationary measure of the nonlinear PDE. Once gain it is interesting to get uniform in times estimation to be able to simulate at fixed time the particles and use them for the evaluation with no loss at each time of the constant in the concentration. Therefore we first prove in the general framework an uniform $T_{1}$ inequality for solution of SDE subject to some convexity at infinity condition. We then show how to use them on our example. 


\subsection{Uniform transportation cost inequality under convex at infinity condition}

Let $X$ be the solution of the following stochastic differential equation:

$$
d X_{t}=d B_{t}+b\left(X_{t}\right) d t
$$

where $B$ is a standard Brownian motion on $\mathbb{R}^{d}$ and $b$ a smooth function from $\mathbb{R}^{d}$ to $\mathbb{R}^{d}$. In the case when $b(x)=x, X$ is the well-known Ornstein-Uhlenbeck process and

$$
\sup _{t \geqslant 0} \mathbb{E}\left[e^{\delta\left|X_{t}^{x}-Y_{t}^{x}\right|^{2}}\right]<+\infty
$$

(where $X^{x}$ and $Y^{x}$ are two independent copies starting both at $x$ ) is finite if and and only if $\delta<1 / 2$, since the law of $X_{t}$ is the Gaussian measure

$$
\mathcal{L} a w\left(X_{t}^{x}\right)=\mathcal{N}\left(x e^{-t}, \frac{1-e^{-2 t}}{2}\right) .
$$

Our aim is to extend this assertions to the case when the drift is confining only outside a compact set, and for non-constant diffusion coefficient, so that $X$ is the solution of

$$
d X_{t}=\sigma(t) d B_{t}+b\left(X_{t}\right) d t
$$

We use here the formalism of transportation cost inequalities in $W_{1}$ distance for which a practical criterion based on the integrability of the exponential of the square of the distance is sufficient [DGW04] (and Bolley-Villani [BV05] or Gozlan [Goz05] for a better evaluation of the constant) and which implies interesting deviation inequalities.

Proposition 5.1 Suppose that there exist $\lambda, A>0$ and $C$ such that, for every $x, y \in \mathbb{R}^{d}$,

$$
(x-y) \cdot(b(x)-b(y)) \leq-\lambda|x-y|^{2}+C, \quad\|\sigma\|_{H S} \leq A
$$

then for any $\delta<\lambda / 2 A$ and any $x \in \mathbb{R}^{d}$,

$$
\sup _{t \geqslant 0} \mathbb{E}\left[e^{\delta\left|X_{t}^{x}-Y_{t}^{x}\right|^{2}}\right] \leq 1+(A d+C+1) e^{\delta(A d+C+1) /(\lambda-2 \delta A)},
$$

where $X^{x}$ and $Y^{x}$ are two independent copies of (12) starting at $x$.

So the law of $X_{t}^{x}$ satisfies a $T_{1}$ inequality with a constant $\mathfrak{C}$ independent of time and initial position.

Recall that a $T_{1}$ inequality with constant $\mathfrak{C}$ for a measure $\mu$ reads as:

$$
W_{1}(\nu, \mu) \leq \sqrt{\mathfrak{C} \operatorname{Ent}(\nu \mid \mu)}
$$

for any $\nu$.

Proof

$\triangleleft$ The process $Z=X-Y$ is a solution of

$$
d Z_{t}=\sigma\left(X_{t}\right) d W_{t}^{1}-\sigma\left(Y_{t}\right) d W_{t}^{2}+\left(b\left(X_{t}\right)-b\left(Y_{t}\right)\right) d t
$$

with initial condition $Z_{0}=0$, where $W^{1}$ and $W^{2}$ are two independent Brownian motions. Itô's formula ensures that

$$
\begin{aligned}
e^{\delta\left|X_{t}-Y_{t}\right|^{2}}-e^{\delta\left|X_{s}-Y_{s}\right|^{2} \leq} & 2 \delta \int_{s}^{t}\left(X_{u}-Y_{u}\right) \cdot\left(b\left(X_{u}\right)-b\left(Y_{u}\right)\right) e^{\delta\left|Z_{u}\right|^{2}} d u \\
& +2 \delta A \int_{s}^{t}\left(d+2 \delta\left|X_{u}-Y_{u}\right|^{2}\right) e^{\delta\left|Z_{u}\right|^{2}} d u+M_{t}-M_{s} \\
\leq & 2 \delta \int_{s}^{t}\left(A d+C+(2 A \delta-\lambda)\left|X_{u}-Y_{u}\right|^{2}\right) e^{\delta\left|Z_{u}\right|^{2}} d u+M_{t}-M_{s}
\end{aligned}
$$


where $M$ is a local martingale with quadratic variation given by

$$
\langle M\rangle_{t}=8 A \delta^{2} \int_{0}^{t}\left|X_{u}-Y_{u}\right|^{2} e^{\delta\left|X_{u}-Y_{u}\right|^{2}} d u .
$$

For every $\delta<\lambda / 2 A$, let us denote by $K$ the quantity

$$
K=\sqrt{\frac{A d+C+1}{\lambda-2 \delta A}} .
$$

For every $x \in \mathbb{R}^{d}$,

$$
\begin{aligned}
\left(A d+C-(\lambda-2 \delta A)|x|^{2}\right) e^{\delta|x|^{2}} & \leq\left(A d+C-(\lambda-2 \delta A)|K|^{2}\right) e^{\delta|x|^{2}} \mathbb{I}_{(|x| \geqslant K)}+(A d+C) e^{\delta K^{2}} \\
& \leq-e^{\delta|x|^{2}}+e^{\delta|x|^{2}} \mathbb{I}_{(|x| \leq K)}+(A d+C) e^{\delta K^{2}} \\
& \leq-e^{\delta|x|^{2}}+(A d+C+1) e^{\delta K^{2}}
\end{aligned}
$$

For $R>0$, introduce $T_{R}$ the first time when $Z$ exits the ball of radius $R$ and define $\alpha_{R}$ the function defined by

Then

$$
\alpha_{R}(t)=\mathbb{E}\left[e^{\delta\left|Z_{t \wedge T_{R}}\right|^{2}}\right]
$$

$$
\alpha_{R}(t)-1 \leq 2 \delta(A d+C+1) e^{\delta K^{2}} t,
$$

so that we may let $R$ go to infinity, and have shown that the exponential moment is finite.

Defining now $\alpha(t)=\mathbb{E}\left[e^{\delta\left|Z_{t}\right|^{2}}\right]$, one gets, for $0 \leq s \leq t$,

$$
\alpha(t)-\alpha(s) \leq 2 \delta(A d+C+1) e^{\delta K^{2}}(t-s)-2 \delta \int_{s}^{t} \alpha(u) d u .
$$

As a consequence, $\alpha$ satisfies the following differential inequality

$$
\alpha^{\prime}(t) \leq-2 \delta \alpha(t)+2 \delta(A d+C+1) e^{\delta K^{2}} .
$$

Denoting by $\beta(t)=\alpha(t) \exp (2 \delta t)$, this implies that,

$$
\beta^{\prime}(t) \leq 2 \delta(A d+C+1) e^{\delta K^{2}} e^{2 \delta t},
$$

and then,

$$
\beta(t) \leq \beta(0)+(A d+C+1) e^{\delta K^{2}}\left(e^{2 \delta t}-1\right) .
$$

Besides, $X$ and $Y$ have the same initial condition so $\beta(0)=\alpha(0)=1$. As a conclusion, we get for all $t \geqslant 0$,

$$
\alpha(t) \leq e^{-2 \delta t}+(A d+C+1) e^{\delta K^{2}}
$$

which achieves the first part of the proof. The final statement follows from [DGW04] Theorem 3.1. An explicit expression of $\mathfrak{C}$ is derived in [Goz05] chapter VII or in Bolley-Villani [BV05]. $\triangleright$

An important consequence of a transportation inequality is that we easily obtain deviation inequality for Lipschitz functions. Indeed for all Lipschitz functions $F$ with $\|F\|_{L i p} \leq 1$ and all positive $r$

$$
\mathbb{P}\left(F\left(X_{t}^{x}\right)-\mathbb{E}\left(F\left(X_{t}^{x}\right)\right) \geq r\right) \leq e^{-r^{2} / \mathfrak{C}} .
$$

Remark that the previous proof extends to the case when the initial law $\mu_{0}$ satisfies

$$
\int e^{\delta|x-y|^{2}} \mu_{0}(d x) \mu_{0}(d y)<+\infty
$$

(that is $\mu_{0}$ satisfies a $T_{1}$ inequality) just choosing two independent variables $X_{0}$ and $Y_{0}$ of law $\mu_{0}$. 


\subsection{A concentration inequality for the stationary measure}

We may now recombine results inherited from the previous sections to get an useful inequality for the evaluation of $\int f d u_{\infty}$ when $f$ is a Lipschitz function. From the previous section, if (A) holds and $\mu_{0}$ satisfies a $T_{1}$ inequality, the particle system satisfies a $T_{1}$ inequality with constant $\mathfrak{C} N$ (for some $\mathfrak{C}$ independent of time), which thus leads to the following:

for $f$ Lipschitz (in $\mathbb{R}^{d}$ ) with $\|f\|_{\text {Lip }} \leq 1$, for all positive $r$ and all $t$

$$
\mathbb{P}\left(\frac{1}{N} \sum_{k=1}^{N} f\left(X_{t}^{k, N}\right)-\mathbb{E} f\left(X_{t}^{1, N}\right) \geq r\right) \leq e^{-N r^{2} / \mathfrak{C}} .
$$

Remark now that for a Lipschitz function satisfying $\|f\|_{L i p} \leq 1$, if the convexity condition $\mathbf{C}(\mathbf{A}, \alpha)$ holds, the uniform propagation of chaos of Theorem 3.2 tells us

$$
\left|\mathbb{E} f\left(X_{t}^{1, N}\right)-\int f(y) u_{t}(y) d y\right| \leq W_{1}\left(u_{t}^{1, N}, u_{t}\right) \leq W_{2}\left(u_{t}^{1, N}, u_{t}\right) \leq\left(\frac{K}{N^{\frac{1}{1+\alpha}}}\right)^{1 / 2}
$$

so that for all $r \geq\left(\frac{K}{N^{\frac{1}{1+\alpha}}}\right)^{1 / 2}$

$$
\mathbb{P}\left(\frac{1}{N} \sum_{k=1}^{N} f\left(X_{t}^{k, N}\right)-\int f(y) u_{t}(y) d y \geq r-\left(\frac{K}{N^{\frac{1}{1+\alpha}}}\right)^{1 / 2}\right) \leq e^{-N r^{2} / \mathfrak{C}} .
$$

We may now use convergence in $W_{2}$ distance of the solutions of the nonlinear SDE towards the stationary measure given in Theorem 4.1 to get

Proposition 5.2 For all $r \geq\left(\frac{K}{N^{\frac{1}{1+\alpha}}}\right)^{1 / 2}+\sqrt{\beta(t)}$,

$$
\mathbb{P}\left(\frac{1}{N} \sum_{k=1}^{N} f\left(X_{t}^{k, N}\right)-\int f(y) u_{\infty}(y) d y \geq r-\left(\frac{K}{N^{\frac{1}{1+\alpha}}}\right)^{1 / 2}-\sqrt{\beta(t)}\right) \leq e^{-N r^{2} / \mathfrak{c}}
$$

where $\beta(t)$ is one of the functions governing the decay of $W_{2}^{2}\left(u_{t}, u_{\infty}\right)$ described in Theorem 4.1.

It is quite hard to imagine to extend to our case uniform result over Lipschitz function (i.e. deviation of the $W_{1}$ distance between the empirical law of the particles and the stationary measure) as in Bolley-Guillin-Villani [BGV05] as it requires a dynamic coupling which can be achieved only for potential $W$ whose Hessian is bounded (in the sense of matrix).

Let us finally note than one can use an Euler-Maryama scheme preserving square exponential integrability and with good stability property [LMS06, Th1 and Th 4] to simulate the particle system leading to the same concentration inequality. Note that the recurrence property needed for the stability of this adaptive scheme in [LMS06] is exactly our condition of convexity at infinity.

\section{References}

[BCCP98] D. Benedetto, E. Caglioti, J. A. Carillo, and M. Pulvirenti. A non Maxwellian steady distribution for one-dimensional granular media. J. of Stat. Physics, 91(5/6):979-990, 1998.

[BCP97] D. Benedetto, E. Caglioti, and M. Pulvirenti. A kinetic equation for granular media equation. Rairo Modél. Math. Anal. Num., 31(5):615-641, 1997.

[BGV05] F. Bolley, A. Guillin, and C. Villani. Quantitative concentration inequalities for empirical measures on non compact spaces. To appear in Prob. Theor. Rel. Fields., 2005. 
[BRTV98] S. Benachour, B. Roynette, D. Talay, and P. Vallois. Nonlinear self-stabilizing processes. I. Existence, invariant probability, propagation of chaos. Stochastic Process. Appl., 75(2):173201, 1998.

[BRV98] S. Benachour, B. Roynette, and P. Vallois. Nonlinear self-stabilizing processes. II. Convergence to invariant probability. Stochastic Process. Appl., 75(2):203-224, 1998.

[BV05] F. Bolley and C. Villani. Weighted Csiszár-Kullback-Pinsker inequalities and applications to transportation inequalities. Annales de la faculté des Sciences de Toulouse, 6:331-352, 2005.

[CL94] P. Cattiaux and C. Léonard. Minimization of the Kullback information of diffusion processes. Ann. Inst. Henri Poincaré, 30(1):83-132, 1994. and correction in Ann. Inst. Henri Poincaré vol.31, p.705-707, 1995.

[CMCV03] J. A. Carrillo, R. J. Mc-Cann, and C. Villani. Kinetic equilibration rates for granular media and related equations: entropy dissipation and mass transportation estimates. Rev. Mat. Iberoamericana, 19(3):971-1018, 2003.

[CMCV06] J. A. Carrillo, R. J. Mc-Cann, and C. Villani. Contractions in the 2-Wasserstein length space and thermalization of granular media. Arch. Rational Mech. Anal., 179(2):217-263, 2006.

[DGW04] H. Djellout, A. Guillin, and L. Wu. Transportation cost-information inequalities and applications to random dynamical systems and diffusions. Ann. Probab., 32(3B):2702-2732, 2004.

[DMT95] D. Down, S. P. Meyn, and R. L. Tweedie. Exponential and uniform ergodicity of Markov processes. Ann. Probab., 23(4):1671-1691, 1995.

[Goz05] N. Gozlan. Principe conditionnel de Gibbs pour des contraintes fines approchées et inégalités de transport. PhD Thesis, Université de Paris 10. Available online via http://tel.ccsd.cnrs.fr/documents/archives0/00/01/01/73/tel-00010173-00/tel00010173-00.pdf, 2005.

[LMS06] H. Lamba, J.C. Mattingly, and A. Stuart. An Adaptive Euler-Maruyama Scheme for SDEs: Convergence and Stability. Preprint. Available online via http://front.math.ucdavis.edu/math.NA/0601029, 2006.

[Mal01] F. Malrieu. Logarithmic Sobolev inequalities for some nonlinear PDE's. Stochastic Process. Appl., 95(1):109-132, 2001.

[Mal03] F. Malrieu. Convergence to equilibrium for granular media equations and their Euler schemes. Ann. Appl. Probab., 13(2):540-560, 2003.

[Mé195] S. Méléard. Asymptotic behaviour of some interacting particle systems: Mc Kean-Vlasov and Boltzmann models. Probabilistic models for non linear partial differential equations. ed. Talay and Tubaro, Lecture Notes in Mathematics 1627:42-95, 1995.

P. Cattiaux: École Polytechnique, CMAP, CNRS 756, 91128 Palaiseau Cedex FRANCE and Université Paris X Nanterre, Equipe MODAL'X, UFR SEGMI, 200 avenue de la République, 92001 Nanterre cedex, FRANCE.

Email: cattiaux@cmapx.polytechnique.fr

A. Guillin: CEREMADE, UMR CNRS 7534, Place du Maréchal De Lattre De Tassigny 75775 PARIS CEDEX 16 - FRANCE. 
Email: guillin@ceremade.dauphine.fr

Web: http://www.ceremade.dauphine.fr/ guillin/

F. Malrieu: IRMAR, Université Rennes 1, Campus de Baulieu, 35042 Rennes cedex, France. Email: florent.malrieu@univ-rennes1.fr

Web: http://name.math.univ-rennes1.fr/florent.malrieu/ 